\title{
L'évolution et l'apport des chemins de fer secondaires en traction électrique et thermique (automotrices et autorails)
}

Yves Machefert-Tassin

\section{CpenEdition}

\section{Journals}

Édition électronique

URL : https://journals.openedition.org/rhcf/2043

DOI : 10.4000/rhcf.2043

Éditeur

Rails \& histoire

Édition imprimée

Date de publication : 2 mai 2002

Pagination : 146-174

ISBN : 00996-9403

ISSN : 0996-9403

Référence électronique

Yves Machefert-Tassin, «L'évolution et l'apport des chemins de fer secondaires en traction électrique et thermique (automotrices et autorails) », Revue d'histoire des chemins de fer [En ligne], 24-25 | 2002, mis en ligne le 13 avril 2015, consulté le 22 avril 2022. URL : http://journals.openedition.org/rhcf/2043 ; DOI : https://doi.org/10.4000/rhcf.2043 


\section{L'évolution et l'apport des chemins de fer secondaires en traction électrique et thermique (automotrices et autorails)}

Les solutions originales utilisées par de grands ingénieurs d'infrastructure en chemins de fer secondaires sont bien connues et parfois reconnues. Il n'en est pas de même en traction, sauf les noms des créateurs ou constructeurs de systèmes complets, tel Decauville, ou de dispositifs pour locomotives à vapeur confrontées avec des courbes de faible rayon, qu'elles soient ou non articulées : ce sont alors, en général, des inventions britanniques, allemandes, ou autrichiennes.

C'est pourquoi il nous a semblé intéressant de retrouver les nombreux apports inventifs, et surtout constructifs et expérimentaux, que les chemins de fer secondaires de France ont apporté à tous les autres, y compris en devançant souvent les applications des mêmes procédés sur les grands réseaux. Certes, les multiples constructeurs ont vu dans les réseaux secondaires le moyen de s'engager, à frais relativement modestes, dans des réalisations nouvelles à risques limités, prototypes pouvant, au mieux, aboutir à des applications à beaucoup plus grande échelle. Nous considérerons ici l'évolution des deux domaines de la traction électrique et des autorails, les ayant particulièrement étudiés, professionnellement, et au cours de participations à des études collectives historiques échelonnées dans le temps ${ }^{1}$. On y constate le rôle particulièrement important des bureaux d'études de constructeurs, stimulés par les problèmes et nécessités d'exploitation très spécifiques à des réseaux ferrés parcimonieux de leurs ressources, sinon impécunieux, mais ne refusant pas les innovations. Certains aidaient d'ailleurs souvent ces mêmes constructeurs, à l'aide de leurs propres ateliers, lors des mises au point du matériel achevé. D’autres essayaient ensuite de réaliser eux-mêmes l'adaptation des éléments moteurs neufs lors de rénovations ou de transformations. Les résultats en furent suffisamment probants malgré leur extrême diversité pour que nous résumions, sans exhaustivité, toutes ces tentatives, ou presque, ayant abouti à des matériels roulants, certes, mais plus ou moins réussis. Ces innovations ont toutefois donné des lettres de noblesse technique à des réseaux 
souvent décriés aujourd'hui pour des raisons économiques. Pourtant, s'ils ont presque tous disparu, sauf quand ils subsistent au titre de patrimoine d'intérêt touristique, c'est par eux que d'éminents services furent rendus à la recherche et que des trouvailles, et la viabilité d'engins moteurs devinrent acceptables par des grands réseaux souvent trop timorés pour s'intéresser à des idées étrangères au sérail.

\section{Les lignes électrifiées (1 600 km en continu, 550 en alternatif)}

Bien que n'ayant représenté, en longueur de lignes, que $10 \%$ environ du total des chemins de fer secondaires (soit $2250 \mathrm{~km}$ sur $23000 \mathrm{~km}$ hors tramways urbains), il est, en vérité, fort difficile de distinguer les chemins de fer secondaires électrifiés des tramways électriques classiques. Nous avons vu qu'il n'existait pas de définition juridique permettant de faire entrer une ligne particulière dans une catégorie définie. Mais nous voyons facilement leurs limites dans les longueurs individuelles des lignes, même si elles sont raccordées en bi-courant ou bi-tension, à des réseaux de tramways urbains existants. Ce qui, soit dit en passant, nous amène à certain étonnement devant la redécouverte des vertus du «tram-train » pratiqué à Karlsruhe, Sarrebruck, ou bientôt Mulhouse, alors que cette forme existait déjà, au début de ce siècle, à Lyon, Grenoble et autres lieux.

Certes, les voies ferrées secondaires électrifiées furent en relatif petit nombre, lequel ne préjuge en rien de leur importance qualitative. En fait, les travaux importants d'installations de lignes aériennes, la construction d'usines génératrices et de sous-stations, l'acquisition de motrices ou de locomotives coûteuses ne pouvaient être en principe envisagés que dans des cas bien déterminés : exploitation intensive de lignes à fort trafic voyageurs, de type suburbain, nécessitant des navettes fréquentes, ou établissement de voies ferrées dans des régions accidentées. Pour ces dernières, les tracés économiques adoptés pour réduire le nombre et l'importance des ouvrages d'art imposaient des courbes de faible rayon et des rampes sévères, excluant l'emploi de la traction à vapeur. Dans les pays de montagne, la « houille blanche », l'énergie hydroélectrique, permettait l'obtention de courant à bon marché, avec le type de courant électrique le plus convenable aux nécessités de traction.

Nous verrons cependant quelques exemples (Loir-et-Cher, réseau du Tarn-Haute-Garonne, voies ferrées départementales du Midi) où aucune de ces raisons n'a été vraiment déterminante dans le choix de l'électricité comme mode de traction. Tout se passe comme si certains départements, pour des raisons de prestige local, avaient tenu à ce 
que leurs voies ferrées d'intérêt local apparaissent dotées de l'équipement le plus moderne, et ce l'était souvent, à l'époque, mais qui n'a pu leur permettre de perdurer de ce seul fait.

Les différents réseaux électrifiés étant répartis de façon assez clairsemée sur la carte des chemins de fer secondaires, ils ne se sont pas souciés de raccordements possibles, et ont adopté le mode d'électrification qui leur paraissait, à l'époque, et compte tenu de leurs exigences particulières, le plus apte à assurer une exploitation satisfaisante. Il en résulte une très grande diversité dans la nature et la tension du courant de traction, le mode de captage (perche, archet, pantographe), ce qui confère un intérêt tout particulier à l'étude des petits réseaux électrifiés. Ceci leur a aussi permis de jouer au mieux la compétition technique entre constructeurs, chacun pouvant essayer des modèles inacceptables par les grands réseaux. Ceux-ci nous apparaissent alors comme autant de lignes expérimentales sur lesquelles les constructeurs purent mettre au point leurs conceptions et, là aussi, les chemins de fer secondaires firent œuvre de pionniers, trop souvent oubliés, particulièrement en France.

Un trait commun et fondamental à tous doit cependant être souligné : l'importance considérable des automotrices dans le parc de traction. Beaucoup de chemins de fer secondaires, à la différence des grands réseaux, ne possédèrent jamais de locomotives et assurèrent la remorque de tous leurs trains (voyageurs et mixtes) uniquement avec des automotrices.

Traction par courant continu à basse tension puis à moyenne tension (500 à 1200 volts)

Ce mode d'électrification a été surtout utilisé par des réseaux ayant un caractère plus spécifiquement « tramway ». Une tension voisine de 600 volts et très souvent une prise de courant assurée par perches à roulettes caractérisent ces lignes, à fil de contact simple le plus souvent.

Un certain nombre de grandes villes possédaient des lignes de tramways s'aventurant fort loin en campagne, quelquefois sur plateforme indépendante et qui prenaient l'aspect de véritables chemins de fer électriques : lignes de Bordeaux à Beychac et de Bordeaux à Camarsac, réseau suburbain de Tours raccordé au réseau à vapeur des C.F.D. d'Indre-et-Loire, lignes de la banlieue de Strasbourg vers Westhoffen, Truchtersheim, Ottrott, Marckholsheim, sur lesquelles étaient effectués en saison d'importants transports de betteraves.

Le réseau départemental des Tramways de Nice et du littoral envoyait des antennes très loin de la côte vers Bendejun, Grave-dePeille, Levens $(23 \mathrm{~km})$. La ligne Menton-Sospel longue de $17 \mathrm{~km}$ qui 
escaladait le col de Castillon par des rampes de $65 \mathrm{~mm}$ par mètre fut supprimée en 1931, mais il en reste des ouvrages d'art remarquables, dont l'importance est sans commune mesure avec le trafic insignifiant que connaissait cette section : deux aller et retour par jour! Ce réseau était exploité par de grosses motrices à bogies de 123 et $177 \mathrm{~kW}$ et des tracteurs à deux essieux ou à bogies assuraient, avec un parc de 160 wagons, le service des marchandises.

Certaines lignes suburbaines de la Société grenobloise de tramways électriques et, en particulier, la ligne de Villard-de-Lans (tension 800 volts en campagne et 600 volts dans la partie urbaine) possédaient le caractère d'un chemin de fer d'intérêt local. Ceci est encore plus vrai du réseau des Voies ferrées du Dauphiné qui, sur la ligne GrenobleUriage-Vizille, assurait, en plus d'un important trafic suburbain de voyageurs, la traction de nombreux trains de chaux destinés aux usines de la vallée de la Romanche, à l'aide de tracteurs électriques de $110 \mathrm{~kW}$.

Le réseau Pierrefitte-Cauterets-Luz dont la première ligne fut construite en 1897 était caractérisé par l'emploi pratiquement systématique d'automotrices, tant pour les voyageurs que pour les marchandises. Ces voitures, le plus souvent dépourvues de remorques, se suivaient en marchant à vue. Le tronçon de Cauterets à La Raillère fut exploité par le matériel d'origine qui gravit gaillardement les rampes de $8 \%$ en adhérence simple jusqu'en 1970 (comme une partie du matériel conservé depuis).

La ligne d'intérêt local de Bellegarde à Chézery, isolée du reste du réseau départemental de l'Ain, fut électrifiée en 600 volts continu dès sa construction en 1913 et dotée de trois automotrices du type tramway montées sur trucks Brill à deux essieux et munies de deux moteurs de $40 \mathrm{~kW}$. Ce parc fut renforcé en 1932 par une automotrice à bogies d'une puissance de $110 \mathrm{~kW}$.

Citons enfin, comme transition vers les tensions élevées, sans utiliser le retour du courant par les rails alors générateurs de difficultés à cause de courants vagabonds dérivés et de l'électrolyse qui pouvait en résulter, la ligne du Havre au Conquet de $24 \mathrm{~km}$, comportant deux fils de contact à 600 et 0 volts, ce dernier assurant seul le retour de courant.

Le courant continu à deux ponts et les tensions supérieures (1 200 à 2400 volts)

L'électrification en courant continu à basse tension ne pouvait cependant être appliquée qu'à des lignes relativement courtes car les pertes en ligne auraient nécessité l'installation de nombreuses sous-stations peu espacées. Dans ces conditions, les frais d'électrification devenaient prohibitifs, compte tenu du faible trafic à espérer. 
Il était difficile d'augmenter la tension car, vers 1900, et même après cette date, l'état de la technique ne permettait guère de s'écarter des moteurs classiques isolés pour 600 volts. Une solution ingénieuse fut trouvée, qui consistait à prévoir deux fils de trolley isolés portés à des tensions égales et opposées, les rails étant évidemment au potentiel zéro, sans rôle conducteur, comme au Havre.

La ligne de Grenoble à Chapareillan, longue de $43 \mathrm{~km}$, fut ainsi mise en service selon ce système à partir de 1899 et exploitée avec de petites motrices pesant $11 \mathrm{t}$ dont les deux moteurs de $26 \mathrm{~kW}$ étaient constamment couplés en série. Deux perches à roulettes placées côte à côte, comme dans d'autres installations en courant alternatif triphasé (Evian, Lugano), assuraient le captage du courant sur les deux fils de trolley parallèles au potentiel de plus 600 volts et moins 600 volts. Ce n'est qu'en 1947 que le tramway de Chapareillan, limité au service Grenoble-Les Aiguinards et devenu un véritable anachronisme, disparut totalement.

Selon le même principe, mais avec des tensions portées à plus ou moins 1200 volts, la ligne de $30 \mathrm{~km}$ de Saint-Georges-de-Commiers à La Mure, jusque-là exploitée avec des locomotives à vapeur, fut électrifiée à partir de 1903 (Thury et Sêcheron-Genève).

Pour y remorquer des convois lourds d'anthracite, les locomotives pèsent 50 puis $60 \mathrm{t}$, avec des puissances de 368 à $500 \mathrm{~kW}$ et ensuite huit automotrices (C.F.T.H.). Prolongée de La Mure à Corps après 1920 ce fut la première ligne à bénéficier du 2400 volts à un seul fil de contact, autorisé en variante au 1500 volts, puis l'ensemble de la ligne y fut ensuite converti. Ce fut aussi une des premières électrifications à bénéficier en sous-station de redresseurs polyanodiques à vapeur de mercure $(\mathrm{BBC})$.

Ces installations complexes à deux fils ont précédé celles, plus classiques, en caténaire à courant continu 1500 volts du réseau basque des V.F.D.M. (1 350 volts en 1917), disparu de 1935 à 1948, et celles de Morez à Nyon (1921), l'étoile de Sirod (V.F.C., 1927) et surtout Annemasse-Samoens (V.F.N., 1932-1959) et Toulouse-Castres (V.F.D.M., 1932-1939) beaucoup trop éphémère. N’a survécu côté suisse, à 2200 volts continu, que la ligne internationale de Morez à Nyon (1921) fermée en 1958 côté France !

\section{Le courant alternatif à haute tension}

L'électrification par courant continu à deux ponts n'offrait qu'une solution compliquée au problème de l'alimentation de lignes étendues, avec le minimum de sous-stations. L'isolement des conducteurs au droit 
des aiguilles et croisements constituait une difficulté indéniable, tout comme en alternatif triphasé. Par ailleurs on désirait utiliser des caténaires. Par conséquent, d'autres réseaux, souvent à l'initiative des constructeurs (Westinghouse, Schneider-Westinghouse, puis Jeumont et S.A.C.M.), préférèrent se tourner vers l'emploi du courant alternatif monophasé à haute tension et, en particulier, du courant à 25 périodes qui pouvait être considéré alors comme le courant industriel dans de nombreuses régions et, une fois de plus, les chemins de fer secondaires firent œuvre de précurseurs, non pas en réalisant des expériences sur des lignes particulières, mais en électrifiant des réseaux entiers en courant industriel quelque quarante ans avant les premiers essais de la SNCF en 50 périodes. Non moins de $550 \mathrm{~km}$ de lignes furent ainsi réalisés, bien avant la règle du continu, de 1910 à 1920 et même après. Dès 1910, les Tramways des Alpes-Maritimes, affluents du réseau des Alpes des Chemins de fer du sud de la France, mettaient en service les lignes de Grasse à Pré-duLac et de la vallée de la Vésubie. Un vaste ensemble d'embranchements électrifiés en 6600 volts se développa autour des artères principales à vapeur de Nice à Meyrargues et de Nice à Digne. Ces lignes souvent mal tracées, de profil difficile (rampes de $90 \%$ ) et desservant des régions à peuplement réduit, et en butte à la concurrence des cars de tourisme, avec un trafic marchandises insignifiant, furent supprimées entre 1928 et 1932. Elles furent exploitées, au début, par 40 petites automotrices de 14 t Decauville montées sur trucks Brill à deux essieux à faible empattement, munis de deux moteurs à répulsion de $20 \mathrm{~kW}$ S.A.C.M. La prise de courant se faisait par archet. Ces voitures fonctionnaient normalement en unités multiples, groupées par deux, et assuraient la traction des trains de voyageurs et mixtes.

En 1914, le parc fut renforcé par six automotrices à bogies (Nivelles et C.G.E.-Creil), équipées de quatre moteurs série de $55 \mathrm{~kW}$ et prenant le courant par pantographe. Elles n'ont pas suffit à augmenter l'attrait d'un trafic peu rémunérateur, et l'expérience ne dura que 22 ans.

Les Tramways électriques du Loir-et-Cher (fig. 1) exploitèrent, à partir de 1911, $153 \mathrm{~km}$ de voies ferrées électrifiées en 6500 volts autour de Blois et Châteaudun avec 12 automotrices Soulé-Jeumont à bogies munies de deux moteurs de $55 \mathrm{~kW}$. La faiblesse du trafic entraina la fermeture du réseau dès 1933-1934, car il était généralement en accotement et gênait l'élargissement des routes départementales concernées. De plus la centrale était thermique, et n'offrait que $750 \mathrm{~kW}$ traction pour 4000 installés. 


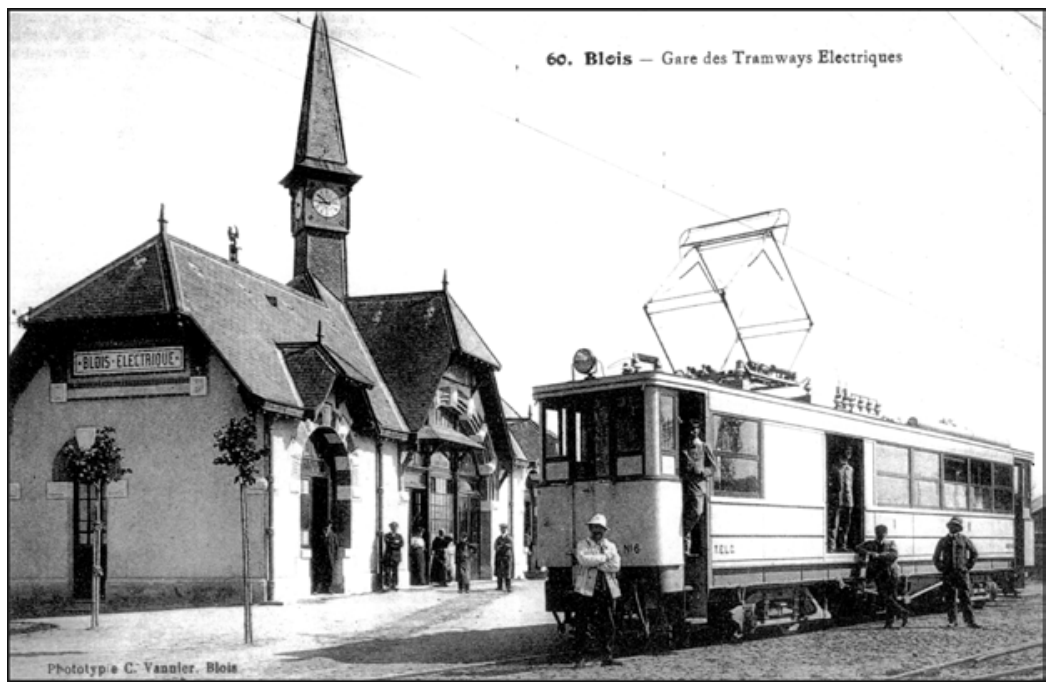

Figure 1 : Automotrice en alternatif 6 kV-25 Hz des Tramways électriques du Loir et Cher (T.E.L.C.) en gare de « Blois-Électrique » qui ont circulé de 1919 à 1934 (construction Soulé-Jeumont).

Coll. Y. Machefert-Tassin, cl. d.r.

Les Chemins de fer départementaux de la Haute-Vienne, équipés en 10000 volts (600 volts pour les sections urbaines dans Limoges), eurent une existence beaucoup plus longue, puisque ce réseau de $345 \mathrm{~km}$, dont la construction débuta en 1911, ne disparut définitivement qu'en 1949. La plupart des lignes de cet ensemble étaient très accidentées, certaines rampes sur Limoges-Peyrat-le-Château atteignaient 80 \%o, sans rail-frein. Le matériel de traction comprenait 21 automotrices « Francobelge » à bogies Brill pesant $28 \mathrm{t}$, munies de quatre moteurs de $40 \mathrm{~kW}$ à collecteur, du type série compensé suisse, et 18 automotrices à deux essieux sur truck Brill comportant deux moteurs du même type. De tels moteurs, à $25 \mathrm{~Hz}$, adaptables au courant $50 \mathrm{~Hz}$, ont ainsi précédé de 40 ans ceux des automotrices SNCF d'Annecy dont nous fêtons le cinquantenaire! Neuf de ces voitures à essieux furent ultérieurement transformées avec bogies et 16 automotrices virent leur puissance portée à $206 \mathrm{~kW}$. La prise de courant se faisait par pantographes. La ligne aérienne à suspension caténaire était portée par des poteaux en béton fabriqués dans les ateliers du réseau. L'alimentation en courant assurée essentiellement par l'usine hydraulique d'Eymoutiers, suffisait largement au réseau et la vente du surplus à d'autres consommateurs, au voisinage 
des lignes, apportait un complément de ressources à la compagnie. Il existe encore un vestige patrimonial de la ligne à l'ancien village d'Oradour-sur-Glane, de triste mémoire.

Les Chemins de fer d'intérêt local du Territoire de Belfort, rayonnant autour de la ville avec une antenne importante aboutissant à Sochaux, furent mis en service en 1913. Le parc traction comprenait des automotrices à bogies à quatre moteurs S.A.C.M. de 26 à $33 \mathrm{~kW}$ du type à répulsion compensé. La ligne était alimentée en 6600 volts. Les installations électriques ayant été détruites au cours de la guerre de 1939-1945, l'exploitation, un moment assurée par autorails diesel sur la ligne d'Étueffont-Haut, fut arrêtée définitivement en 1948.

La Société des chemins de fer des Pyrénées-Orientales construisit en 1913 son réseau de montagne reliant Arles-sur-Tech à Saint-Laurent-de-Cerdans et à Prats-de-Mollo. Ces lignes à voie de $1 \mathrm{~m}$, comportant des rampes sévères de plus de $5 \%$ et des rayons inférieurs à $20 \mathrm{~m}$, étaient électrifiées en 6000 volts d'origine hydraulique. Quatre automotrices ACNE-Jeumont de $28 \mathrm{t}$ munies de quatre moteurs sérierépulsion type Latour de $40 \mathrm{~kW}$ pouvant remorquer 40 à $70 \mathrm{t}$, assurèrent le service jusqu'en 1937.

Les Chemins de fer de la Camargue, désireux de réduire leurs frais d'exploitation et aux prises avec des difficultés d'alimentation de leurs machines à vapeur en raison de la qualité des eaux disponibles, électrifièrent leurs lignes de Nîmes à Arles et Saint-Gilles dès 1920 et le reste du réseau (ligne du Delta) en 1932. La traction était assurée par un parc de 19 automotrices Schneider-Westinghouse (S.-W.) de $235 \mathrm{~kW}$ aux moteurs fonctionnant sous 6600 volts à la caténaire. Certaines comportaient un compartiment à voyageurs. Le profil facile (rampes inférieures à $12 \%$ ) permettait des vitesses commerciales assez élevées : $30 \mathrm{~km} / \mathrm{h}$ sur la ligne d'Arles aux Saintes-Maries-de-la-Mer. La mise en service de remorques allégées en 1949 et l'existence d'un important trafic de sel marin à destination des industries chimiques des Alpes, sur la ligne de Salins-de-Giraud, n'ont pu empêcher la disparition totale de ce réseau en 1958, qui fut un des derniers à être exploité en traction électrique, mais concurrencé par un récent embranchement voie normale SNCF, sans transbord du sel.

La Régie départementale des tramways de l'Ain, après quelques essais effectués en 1926 sur le tronçon Préau-Saint-Martin-du-Fresne avec l'assistance du groupe Giros, Loucheur et Cie (qui avait construit les Chemins de fer départementaux de la Haute-Vienne), électrifia selon le même système : 10000 volts, 25 périodes, les lignes Nantua-Hauteville en 1931, Saint-Martin du Fresne-Ambérieu et Préau- 
Cerdon en 1933. Six automotrices Jeumont de $92 \mathrm{~kW}$ et quatre locomotives Jeumont de $184 \mathrm{~kW}$ assuraient les services voyageurs et marchandises de ces lignes dont les rampes atteignaient $50 \%$. Ce matériel d'aspect moderne ne fit pas une bien longue carrière puisque la dernière ligne des Tramways de l'Ain fut supprimée en 1954.

Nous pouvons également mentionner rapidement quelques réseaux intéressants équipés en alternatif, mais cette fois à la fréquence $162 / 3 \mathrm{~Hz}$, tout comme l'était le réseau du Midi avant 1920 :

- lignes suburbaines de Lyon à Montluel et Lyon à La Balme électrifiées en 6000 volts.

- Lourdes à Bagnères-de-Bigorre et Gripp-Artigues. Ces $41 \mathrm{~km}$ de lignes exploités par une société filiale du réseau du Midi (alors en cours d'électrification en $162 / 3 \mathrm{~Hz}$ ), les Voies ferrées d'intérêt local des Pyrénées, furent inaugurées en 1914 et supprimées en 1937. La tension était de 6000 volts.

Les Tramways électriques du Libournais étaient électrifiés en 6600 volts. Mis en service en 1913, ils subsistèrent jusqu'en 1952. Le matériel comprenait deux tracteurs à marchandises à quatre moteurs de $40 \mathrm{~kW}$ du type répulsion-série-répulsion et cinq automotrices à voyageurs munies seulement de deux moteurs identiques (voir S.A.C.M.-Belfort).

Enfin, beaucoup plus récemment, de 1936 à nos jours, signalons le cas exceptionnel des Houillères du bassin de Lorraine sur ses lignes de Faulquemont et Folschviller, à l'origine à 1500 volts avec locomotives allemandes, transformées en 1952 directement à 25000 volts et $50 \mathrm{~Hz}$, les deux engins de traction recevant pour leur part un équipement redresseur à partir de l'alternatif, utilisant les premiers ignitrons français S.-W. destinés aux BB 12000 de la SNCF en 1954... Les lignes ont fini avec les mines après 1970.

\section{Cas spéciaux d'adaptation électrique}

Outre les cas d'utilisation directe du courant triphasé, essayé à Évian par Ganz (1898), qui n’ont concerné en France que les deux lignes pyrénéennes de Bagnères-de-Luchon à Superbagnères et de SaintIgnace à la Rhune, toutes deux en voie métrique et courant $3 \mathrm{kV}$ à 40 périodes (puis $50 \mathrm{~Hz}$ ) et matériel moteur interchangeable (SLMBBC-1912-1914-235 kW) on connait aussi un cas très extraordinaire de ligne passée du pétroléo-électrique à l'électrique. Il s'agit de la ligne de 4,5 km des houillères de Carvin à Libercourt (1865-1985). Exploitée dès 1911 avec des autorails benzoélectrique (Westinghouse) puis Renault K en 1923, la ligne est électrifiée en 1935 en 700 volts alternatif, 
50 périodes par transformation des autorails Renault en automotrices électriques avec matériel Jeumont dérivé des tramways de l'Ain. Mais la ligne (avec 21 aller-retour par jour !) ne vivra ainsi que jusqu'en 1940.

\section{Traction électrique par voitures à accumulateurs}

Il nous faut enfin ajouter quelques mots d'un mode particulier d'électrification qui prit une certaine importance sur les chemins de fer secondaires : l'emploi d'automotrices à accumulateurs, avec une avance considérable, certes issue des tramways, sur d'autres techniques d'alors.

Certains réseaux, pour lutter contre la concurrence routière, envisagèrent, vers 1925, de séparer radicalement leur trafic marchandises de leur trafic voyageurs et d'utiliser, pour ce dernier, des automotrices électriques à accumulateurs. Ils bénéficiaient, dans ces conditions, des importantes subventions de l'Etat, pouvant dépasser la moitié des dépenses d'installation, prévues pour les réseaux électrifiant leurs lignes, sans pour autant avoir à faire les frais d'équipements coûteux (lignes aériennes, feeders et sous-stations) quelquefois hors de proportion avec le trafic. De plus, la consommation nocturne d'énergie de recharge des accumulateurs, même à mauvais rendement $(50 \%)$ n'était pas coûteuse, venant du réseau général $50 \mathrm{~Hz}$ (ou 25 !).

Dans les années 1927-1931, les Chemins de fer économiques des Charentes mirent en service 15 lourdes voitures à bogies munies de deux moteurs électriques de $40 \mathrm{~kW}$, portant 8,5 td'accumulateurs (fig. 2). La caisse avait été construite par Carel et Fouché et l'équipement réalisé par la S.A.C.M. Ces voitures étaient dotées d'une autonomie de marche de $150 \mathrm{~km}$. Des postes de recharge des batteries, équipés de redresseurs à vapeur de mercure (ampoules Hewittic), étaient installés en divers endroits du réseau : Angoulême, Blanzac, Barbezieux, etc. Ces automotrices pouvaient atteindre seules une vitesse de $42 \mathrm{~km} / \mathrm{h}$ et remorquer, à allure plus réduite, une ou deux voitures du parc, pesant 5 t. En 1929 le parc de ces voitures finit par atteindre 16 unités. Les Chemins de fer du Morbihan, rattachés également au groupe Jeancard, possédèrent six automotrices semblables à partir de 1931. Certaines d'entre elles roulaient encore après 1944.

De leur côté, les Chemins de fer du Sud-Ouest, après l'essai de trois prototypes (1929), exploitèrent leur ligne de Toulouse à Boulognesur-Gesse à l'aide de six automotrices à accumulateurs conçues par De Dion-Bouton. Ces lourdes voitures (34 t avec les batteries) équipées de quatre moteurs de $30 \mathrm{~kW}$ (Brissoneau) remorquaient des voitures à bogies allégées. Elles assurèrent le service de la ligne de Boulogne-surGesse jusqu'à sa fermeture, en 1949, remorquant même à l'occasion des trains de marchandises. 


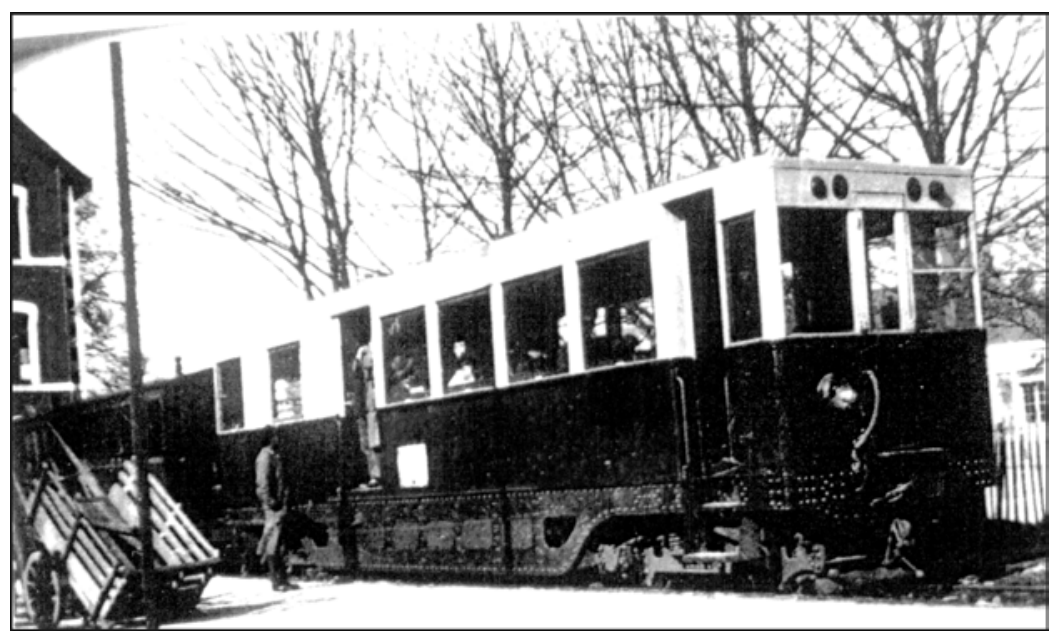

Figure 2 : Automotrice à accumulateurs du réseau des Charentes (1927). Coll. Y. Machefert-Tassin, cl. F.A.C.S.

\section{Conclusion sur la traction électrique}

Une extrême diversité dans les systèmes adoptés, des techniques souvent hardies et parfois très innovantes et des performances honorables ont caractérisé l'électrification des réseaux secondaires. Il peut paraitre surprenant que tout cela ait abouti à une disparition totale des voies ferrées d'intérêt local françaises électrifiées (sauf La Mure et La Rhune), alors que quelques réseaux rarement à traction à vapeur et le plus souvent « diésélisés » subsistent encore en exploitation touristique. Il n'est pas douteux que, dans la plupart des cas, les frais importants des installations électriques n'étaient pas justifiés par les deux ou trois aller et retour quotidiens qui figuraient à l'horaire de la plupart de nos lignes secondaires. Cependant, en quelques cas de sites et de profils difficiles, nos voisins helvétiques ont montré et démontrent encore qu'avec des volontés régionales d'équilibrer les modes de transport et leur aide financière, de nombreuses lignes électrifiées, pas seulement touristiques ou saisonnières, arrivent non seulement à survivre mais à prospérer. Ici encore, notons au passage que ces lignes, ou compagnies privées, servent souvent de précurseurs ou de banc d'essai pour de nombreuses nouveautés techniques que n'osent pas aborder immédiatement les grands réseaux voisins. 


\section{Locotracteurs et locomotives à moteurs à combustion interne}

L'idée d'appliquer le moteur à combustion interne à la traction des convois sur voie ferrée est de celles qui se sont très tôt présentées à l'esprit des constructeurs de matériel roulant.

Outre le médiocre rendement moyen de la machine à vapeur, multipliable avec le diesel par quatre ou cinq fois, la meilleure continuité du couple moteur était favorable à une bonne adhérence. Côté alimentation, le combustible liquide simplifiait les installations fixes, et l'eau n'était plus un problème. De plus, la transmission d'effort moteur aux essieux peut être assurée soit par des dispositifs mécaniques (embrayage et boîte de vitesse), électriques (génératrice et moteur électrique) ou hydrauliques, et tous ont été essayés, voir combinés.

En fait, les premiers engins mis en service furent surtout des tracteurs de chantier à voie de $0,50 \mathrm{~m}$ ou $0,60 \mathrm{~m}$. Au cours de la guerre de 1914, de nombreux locotracteurs furent utilisés sur les chemins de fer de campagne, où ils présentaient l'intérêt particulier de ne donner lieu à aucune lueur ou fumée susceptibles de trahir leur présence (locotracteurs Schneider à voie de $0,60 \mathrm{~m}$ à transmission mécanique et Crochat à voie normale, à transmission électrique, plus particulièrement destinés à la traction des canons lourds de l'A.L.V.F.). Ces engins militaires pouvaient trouver d'autres applications dès 1919, que ce soit en voie normale ou en voie étroite, surtout sur de petits réseaux à faible parc moteur.

\section{Premières réalisations sur les chemins de fer secondaires}

Cependant, malgré les bons résultats obtenus au cours de la guerre, les réseaux secondaires qui, comme nous le verrons plus loin, ont mis rapidement en service, après 1920, de nombreuses automotrices à moteurs à combustion interne, hésitèrent longtemps avant de remplacer leurs locomotives à vapeur.

D'autres auteurs ont pu vous présenter les raisons avancées pour expliquer les retards de modernisation de ce type de traction mais, curieusement, la pression des constructeurs en vue d'obtenir des commandes de redémarrage industriel après 1914-1918, ne concerna que les transports de voyageurs et les autorails, à cause des tendances et demandes des grands réseaux. C'est pourquoi nous verrons les innovations et essais en tout genre concerner avant tout ce type de matériel moteur dès 1920, et non la substitution aux locomotives à vapeur, réalisée bien plus tard.

Par exemple, en dehors de l'usage direct de « surplus » militaires « à pétrole », Schneider ou Crochat, vers 1924, la Régie départementale des tramways de l'Ain construisit dans ses ateliers un petit tracteur à 
partir d'un châssis de wagon. Un moteur « semi-diesel » Ballot de $60 \mathrm{ch}$ $(44 \mathrm{~kW})$ à deux cylindres actionnait une génératrice de $40 \mathrm{~kW}, 220$ volts à excitation indépendante, deux moteurs de traction commandaient les deux essieux par chaînes. Cette machine de $15 \mathrm{t}$ pouvait remorquer $40 \mathrm{t}$ à $40 \mathrm{~km} / \mathrm{h}$ en palier; elle fut utilisée quelque temps sur la courte antenne Pont-de-Vaux-Fleurville. C'était déjà un premier pas vers le diesel-électrique.

En 1925, un tracteur-fourgon Polar-Dewa, de construction suédoise, fut mis en service sur la ligne du Vexin, Valmondois-Marine, des Chemins de fer économiques. Cet engin monté sur trois essieux dont deux moteurs avait un poids de $18 \mathrm{t}$ dont $12 \mathrm{t}$ de poids adhérent et était muni d'un moteur diesel Polar de 75/90 ch, actionnant une génératrice à courant continu de $110 \mathrm{~V}$ alimentant les deux moteurs de traction, totalisant $60 \mathrm{~kW}$.

Ces deux prototypes eurent une courte carrière, mais ont soulevé l'intérêt de divers réseaux.

En 1933, la Régie départementale des tramways de l'Ain mit en service deux locotracteurs diesel-mécaniques, Unic-Codra, qui furent utilisés jusqu'à la fermeture du réseau non électrifié.

A l'occasion de la rénovation du réseau du Var, peu avant 1939, les Chemins de fer de Provence firent construire par Brissonneau et Lotz deux tracteurs fourgons diesel-électriques d'une conception très analogue à celle des autorails du même constructeur. D'un aspect très moderne, pesant $25 \mathrm{t}$, munis de deux moteurs Berliet, leur puissance de $210 \mathrm{~kW}$ leur permettait de remorquer $100 \mathrm{t}$ en palier à $60 \mathrm{~km} / \mathrm{h}$ (effort de traction de $60 \mathrm{kN}$ ). Il est remarquable que certains de ces engins, y compris ceux reconditionnés en Suisse par les Chemins de fer du Jura, connaissent encore un allongement de vie, après plus de 60 ans!

\section{Transformation de locomotives à vapeur en tracteurs diesel}

Le remplacement, vers 1930, des locomotives à vapeur encore conservées après la mise en service des autorails par des locotracteurs, pouvait permettre des économies, à condition toutefois que le prix d'achat de ces derniers ne soit pas trop important, d'où des solutions spécifiques aux chemins de fer secondaires.

Dès 1937, la Compagnie des chemins de fer départementaux transforme en ses ateliers une de ses locomotives à vapeur en tracteur léger. Les résultats ayant paru encourageants, ces transformations furent poursuivies pendant les années suivantes, et étendues à une vingtaine d'unités. De la locomotive à vapeur, le plus souvent à trois essieux couplés, on ne conservait que le châssis, quelquefois raccourci, la suspension, les essieux moteurs, les bielles d'accouplement, le 
tamponnement, l'attelage et la timonerie de frein ; tout le reste, chaudière et mécanisme, était démonté (nous en avons vu à Romorantin lors de notre colloque).

Ces locotracteurs ont été le plus souvent munis de moteurs Berliet de $110 \mathrm{~kW}$ ou Willeme de $144 \mathrm{~kW}$. Leur poids ne dépasse guère $20 \mathrm{t}$, mais la charge remorquée est de l'ordre de 100 t sur des lignes à profil moyen. On estimait en 1947 à environ 50 \% l'économie par rapport à la traction à vapeur, et ce serait encore beaucoup plus de nos jours, même comparé à une chauffe au fuel lourd. Quelques engins de ce type, dont beaucoup proviennent des Ateliers C.F.D. de Montmirail, sont encore en service.

Des locotracteurs analogues, obtenus également par transformation d'anciennes locomotives à vapeur, ont été conçus par d'autres compagnies.

En 1948, la Compagnie générale des voies ferrées d'intérêt local construisit dans ses ateliers de Lumbres et Bapaume deux prototypes : l'un à voie métrique pesant $18 \mathrm{t}$, l'autre à voie normale de $21 \mathrm{t}$, munis du même moteur Willeme de $132 \mathrm{~kW}$, qui furent suivis d'autres réalisations.

La Société générale des chemins de fer économiques mit en service, en 1950, sur le tronçon betteravier à voie de $1 \mathrm{~m}$ de Rogenvilliers à Jouy-le-Châtel (S.-et-M.) deux locotracteurs de 148 kW obtenus à partir de deux anciennes locomotives 031 Cail ; le bissel a été cette fois conservé pour améliorer la tenue de voie. Les Voies ferrées du Dauphiné possédaient aussi cinq locotracteurs « réseau », dont trois à voie normale obtenus par refonte radicale de châssis de locomotives à vapeur 030 à voie métrique!

Mentionnons encore en 1937 le très curieux tracteur diesel-électrique de 22,5 t muni d'un moteur diesel de $160 \mathrm{~kW}$ et d'un mécanisme à faux essieu, construit par Brissonneau et Lotz sur le châssis d'une 030 Blanc-Misseron et qui a assuré pendant plus de 30 ans la remorque de wagons à voie normale sur trucks porteurs évitant les transbordements sur le court embranchement des Usines de Melle, dernier vestige du réseau des Tramways des Deux-Sèvres (3 km, 1901-1975).

\section{Locomotives diesel type USA : une occasion manquée pour beaucoup}

Les surplus de la guerre 1939-1945 ont fourni à plusieurs réseaux à voie normale l'occasion peu coûteuse de renouveler leur parc de traction. En 1946, le réseau de la Gironde de la Société générale des chemins de fer économiques rachète neuf locomotives diesel électriques General Electric type BB USA, de 68 t, munies de deux moteurs Cummins de $368 \mathrm{~kW}$ ce qui permet de remplacer les locomotives à 
vapeur sur les lignes traversant les forêts des Landes. Depuis 1951, le réseau de l'Hérault de la même Société possédait six locomotives BB COFERNA diesel-électriques de $370 \mathrm{~kW}$ également équipées de bogies et de groupes électrogènes américains G.E. Notons que, de nos jours, et 55 ans plus tard, certaines des locomotives G.E. sont encore en état de marche... au Japon (en voie métrique), et ailleurs.

\section{Réalisations ultérieures de locomotives}

Parmi les réalisations des années 1950, sur base d'avant-guerre (voir réseau du Var), il convient de citer la série de dix remarquables locomotives diesel-électriques Brissonneau et Lotz dont initialement quatre exemplaires furent attribués aux Chemins de fer de Provence, quatre aux Voies ferrées du Dauphiné et deux à la Corse.

Ces machines pesant $46 \mathrm{t}$ (50 $\mathrm{t}$ avec lest) sont pourvues de deux groupes électrogènes autonomes de $220 \mathrm{~kW}$, sur deux bogies genre Brill, très adaptés aux voies médiocres. Elles ont assuré les marchandises sur les lignes de montagne Nice-Digne et Bastia-Ajaccio, et certaines ont été rénovées il y a peu.

Citons encore parmi des engins proches de ceux des grands réseaux, les locomotives à voie normale du type 030, diesel-hydrauliques, des Chemins de fer de l'est de Lyon. Ces machines de $54 \mathrm{t}$, pouvant fonctionner accouplées par deux, étaient munies d'un moteur Saurer de $330 \mathrm{~kW}$ et remorquaient des trains de marchandises de $800 \mathrm{t}$ dans la banlieue lyonnaise.

Enfin, en 1960, la Compagnie de chemins de fer départementaux a fait construire dans ses ateliers et chez F.L.C. des locomotives BB de $300 \mathrm{~kW}$ et $32 \mathrm{t}$, dont chaque bogie moteur a ses essieux couplés par bielles ; la transmission hydro-mécanique du type "Asynchro C.F.D. » comporte un coupleur hydraulique, un embrayage-frein, une boite de vitesses à huit rapports et une roue libre, avec un moteur diesel par bogie. Vint-quatre de ces locomotives seront d'ailleurs choisies par la SNCF en 1965 pour constituer la série 71000 , de $400 \mathrm{~kW}$ et $55 \mathrm{t}$, disparue aujourd'hui ainsi que cette solution mécanique complexe et fragile. Des machines analogues ont été en service sur les lignes de la Corrèze, après le réseau C.F.D. du Vivarais. C'est pourquoi, à la fin des années 1960, en avance sur la SNCF, les locomotives à vapeur ont presque totalement disparu du parc de traction des chemins de fer secondaires et les locotracteurs les ont remplacées partout, à l'exception du réseau métrique breton $\mathrm{SNCF}$, alors fidèle, encore pour un temps, à ses puissantes Mallet 030-030 et à ses rapides 230 . 
Il est vrai que la mise en service assez tardive de ces engins diesel, à l'époque du déclin de la plupart des réseaux secondaires, n’a pas vraiment intéressé les constructeurs, sauf les ateliers des principaux groupes, et n'a pas offert de nouveautés aussi marquantes qu'en autorails.

\section{Les automotrices à moteurs thermiques et autorails}

L'emploi d'automotrices pour assurer le service voyageurs sur les chemins de fer secondaires non électrifiés a pris depuis les années 1920 un caractère général et, surtout, a assez largement devancé, que ce soit en technique ou en champ d'application relatif, les grands réseaux, dont l'intérêt pour ces engins fut postérieur, et motivé par les premiers résultats obtenus sur les chemins de fer secondaires, où les moteurs à combustion interne ont pu évincer presque totalement les trains légers de voyageurs ou mixtes à vapeur.

\section{Les précurseurs - vapeur et air comprimé}

Il nous faut remonter bien avant la guerre de 1914, au début du siècle, pour voir apparaittre les premières automotrices sur les chemins de fer secondaires, mais leur entrée en scène se fit bien timidement.

Des lignes ou embranchements de lignes de faible importance furent pratiquement, dès leur ouverture, exploités par des automotrices à vapeur complétant un parc classique restreint.

Dès 1879 le court tronçon à voie de $1 \mathrm{~m}$ reliant Marlieux, station P.L.M. sur la ligne Bourg-Lyon, à la petite ville de Châtillon-surChalaronne voyait circuler une automotrice à vapeur à bogies, munie d'une chaudière Field. D'aspect moderne pour l'époque, construite par Brunner frères à Winterthur, cette voiture avait été rachetée presque neuve à la ligne suisse de Lausanne-Echallens où elle roula fort peu de temps. Après avoir assuré le service concurremment avec les locomotives à vapeur, elle fut convertie en remorque peu après 1914.

En 1899, le tramway de Versailles à Maule mit en service deux « automobiles à vapeur» Pinguély, constituées par une petite locomotive à deux essieux moteurs munie d'une chaudière verticale Field (fig. 3). La caisse renfermant les compartiments à voyageurs reposait à l'avant sur la partie motrice à laquelle elle était articulée par une cheville ouvrière.

Les Établissements Pinguély fournirent en 1903-1905 aux Tramways de l'Indre trois automotrices de conception analogue du type à trois essieux qui circulèrent en particulier sur Argenton-Le BlancChaillac, et sont désignées comme « voitures automobiles ». 
Revue d'histoire des chemins de fer 24-25 (printemps - automne 2001)

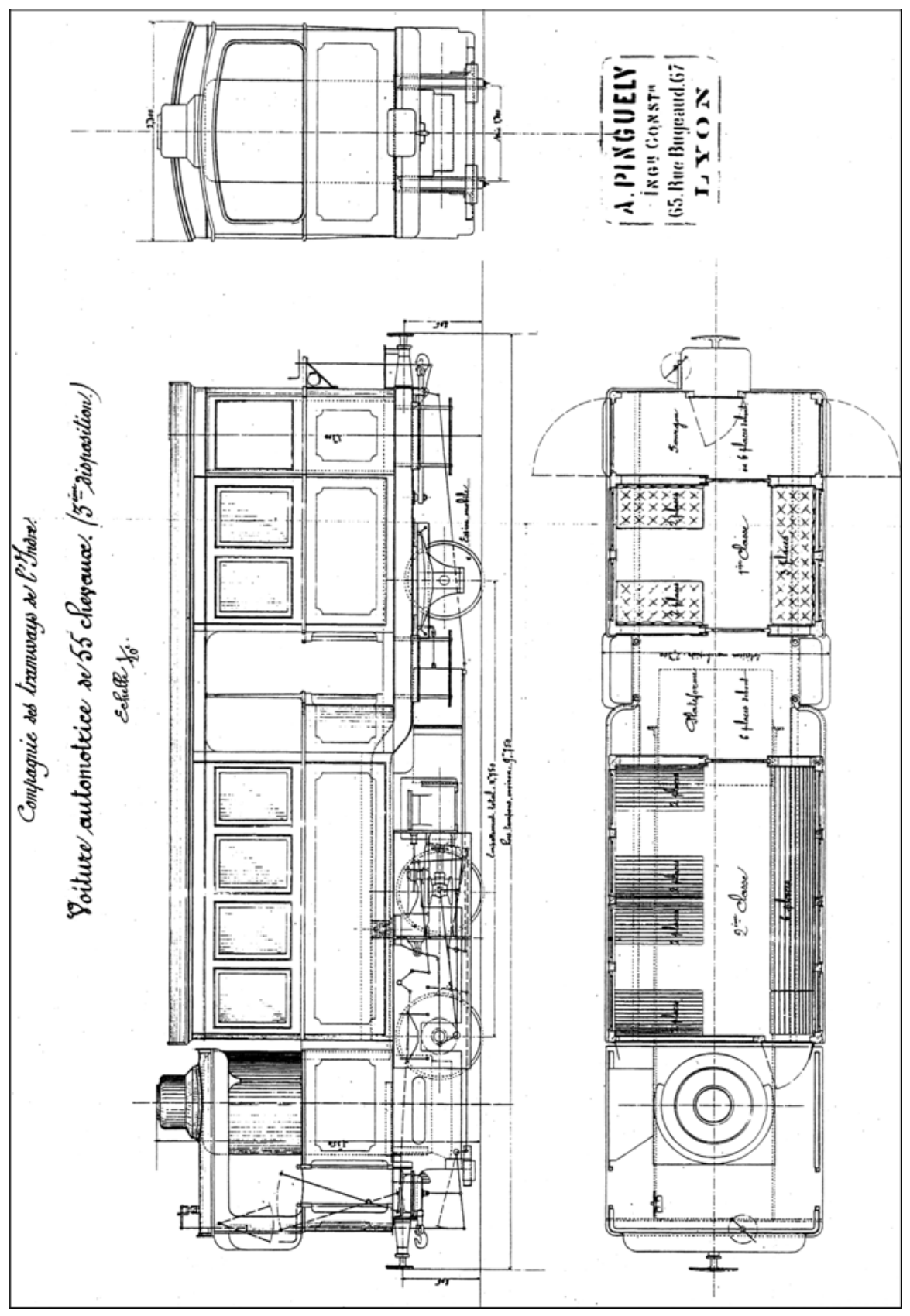

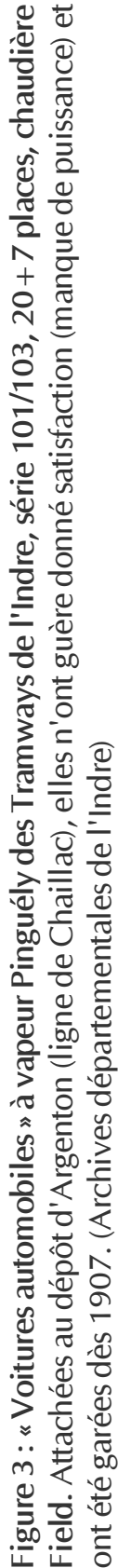


Ces engins, dont le diagramme figurait à l'exposition sur les chemins de fer secondaires berrichons présentée par les Archives départementales de l'Indre lors de notre colloque, comportaient une même disposition : soit, à l'avant, une partie motrice à deux essieux style locotracteur à vapeur, prolongée par une caisse voyageurs de 17 places, le tout, long de $9,75 \mathrm{~m}$, étant porté à l'arrière par un troisième essieu à orientation radiale, pour les courbes de faible rayon. Cette disposition originale n'a pas empêché ces engins d'avoir une douteuse réputation d'insuffisance de puissance, et de vitesse, sur les lignes de l'Indre à profil un peu accentué. Bien que l'idée fût excellente, la réalisation ne fut donc pas à la hauteur des espoirs et ralentit certainement, du moins momentanément, l'extension du système, sans réduire pour autant, au contraire, la diversité des techniques de chaudières ou de moteurs essayés par ces ancêtres des autorails.

Les Chemins de fer du sud de la France (réseau de l'Isère) mirent en service vers 1909-1910, sur le tronçon La Tour-du-Pin-Les Avenières, des automotrices à vapeur caractérisées par la séparation du mécanisme et de la semi-remorque. D'un poids de 19 t et d'une capacité de 32 places pour une consommation de $5 \mathrm{~kg}$ de charbon par $\mathrm{km}$, ces voitures donnèrent de bons résultats haut-le-pied mais, l'augmentation du trafic obligeant à leur faire remorquer une voiture et souvent un wagon, la puissance de $95 \mathrm{~kW}$ devint rapidement insuffisante.

D'autres réseaux firent appel à des voitures réunissant à nouveau sur un même châssis mécanisme et compartiments à voyageurs et bagages.

Depuis 1900 circulaient sur de nombreux réseaux urbains et, en particulier, à Paris, des automotrices à vapeur à très faible empattement, munies de chaudières Purrey comportant deux collecteurs cylindriques réunis par des tubes d'eau placés dans le foyer et des colonnes de retour d'eau extérieures au foyer. Ces voitures robustes, souples et d'entretien moins délicat, malgré les hautes pressions et températures utilisées, que leurs contemporaines, telles celles pourvues de la chaudière Serpollet, furent adoptées par quelques compagnies de chemins de fer d'intérêt local. C'est ainsi que les Chemins de fer du sud de la France (réseau de la Côte-d'Or) utilisèrent deux automotrices Purrey et que les Tramways du Loiret renforcèrent leur service dans la banlieue d'Orléans par des navettes effectuées avec un véhicule analogue. Le service urbain de la ville de Soissons fut assuré par les Chemins de fer de la banlieue de Reims avec deux automotrices Purrey à partir de 1911. Les deux courtes antennes à faible trafic, l'Argentière-La Croisette et Ruoms-Vallon, des Tramways de l'Ardèche furent exploitées dès l'ouverture, en 1912, par des automotrices du même type. On en utilisa également sur le réseau 
des Tramways de Saumur et extensions ainsi que sur le chemin de fer de la vallée de Celles. Tout ceci amena les grandes compagnies à s'y intéresser aussi.

Mentionnons encore les essais, effectués en 1909 sur le réseau de la Côte-d'Or (lignes de Dijon à Saint-Seine-l'Abbaye et de Dijon à Champlitte), d'une automotrice du type Scotte, construite par les Établissements Pétolat. Munie d'une chaudière Field et d'un moteur compound à quatre cylindres, cette voiture atteignait $30 \mathrm{~km} / \mathrm{h}$. Elle comportait un compartiment-fourgon et pouvait transporter 18 voyageurs. Il ne semble pas que ce prototype ait été retenu par le réseau peut-être à cause de sa capacité médiocre - voir Tramways de l'Indre - et des performances insuffisantes.

Vers la même époque, le tramway du Pont-de-Beauvoisin (Savoie) préfère utiliser quelque temps une automotrice à air comprimé du type Mekarski, qui était très en faveur sur beaucoup de réseaux de tramways urbains (Paris, Nantes, Saint-Quentin, etc.). Il est possible que cette automotrice ait été cédée par le réseau voisin des tramways d'Aix-lesBains alors exploité selon ce système. Il est vraisemblable que la nécessité de posséder une usine de compression d'air et des postes de recharge n'était pas sans créer de sérieuses difficultés d'exploitation à un petit réseau isolé, à faible densité de trafic, d'où l'abandon du système sur lignes locales ou secondaires.

\section{Apparition du moteur à combustion interne, du pétrole au diesel}

Dans les dernières années du XIX ${ }^{\mathrm{e}}$ siècle, les premières automobiles commencent à apparaitre un peu partout et peu à peu le moteur à essence ou à pétrole raffiné et la transmission par boîte de vitesses s'imposent. Les réseaux secondaires, jusque-là fidèles à la classique traction à vapeur n'en voient pas la nécessité, avant 1914, sauf exceptions. C'est à peine si l'on peut mentionner (hormis les mines) quelques réalisations d'engins à faible puissance, qui n'étaient en fait que de grosses draisines. Le petit tramway à voie de $0,60 \mathrm{~m}$ desservant le golf de Paris-Plage employait des automotrices Campagne de 30 ch à deux essieux moteurs commandés par chaînes et boite à trois vitesses. Pour être complet, il conviendrait encore de citer la courte ligne à voie de $0,60 \mathrm{~m}$ qui longeait la plage de La Baule, où roulaient de légères automotrices ouvertes, tirant une remorque, la ligne forestière de la Coubre à l'origine en voie de $1 \mathrm{~m}$ exploitée par quatre draisines en raison des dangers d'incendie, et l'éphémère tramway "urbain » de Montargis, dont la guerre de 1914 empêcha la mise en service régulier alors qu'il venait juste d'être terminé. 
En fait, si la transmission de l'effort moteur par l'intermédiaire d'un ensemble embrayage-boite de vitesses donnait satisfaction pour des véhicules automobiles légers, il ne pouvait en être de même avec des engins sur voie ferrée d'un poids beaucoup plus élevé et les constructeurs, qui avaient entrevu le problème lors de la mise en service de camions lourds, restaient forts réservés, l'état de la technique n'autorisant pas alors de grands espoirs dans la transmission mécanique. C'est ce qui explique le succès des automotrices dites " pétroléo-électriques » comportant un moteur à combustion interne actionnant une dynamo qui fournissait le courant à des moteurs électriques de traction du type tramway, préconisées par les constructeurs électriciens de tels systèmes, très cohérents, rendement mis à part.

En 1911, le Tramway de Dinard à Saint-Briac, qui avait déjà utilisé de 1903 à 1908 deux automotrices à vapeur Turgan pour assurer le trafic hors saison, acquit une automotrice Westinghouse à transmission électrique. Cette lourde voiture à bogies (poids en charge $22 \mathrm{t}$ ) était munie d'un moteur à essence de $66 \mathrm{~kW}$. D'une longueur de 12,20 m et capable de transporter cinquante voyageurs, elle se révéla particulièrement gourmande en carburant (100 litres aux $100 \mathrm{~km}$ quand elle fut livrée, consommation qui s'éleva rapidement après $10000 \mathrm{~km}$ à la valeur catastrophique de 200 litres aux $100 \mathrm{~km}$ !). Des automotrices Westinghouse de conception analogue furent mises en service sur les lignes à voie normale de Caen à la mer et de Carvin à Libercourt ainsi que sur la ligne de Versailles à Meulan, mise à voie normale lors de sa reprise par les Chemins de fer de grande banlieue.

Enfin, lorsque l'usure des machines à vapeur sans foyer du système Lamm et Francq, qui assuraient la traction des trains sur la ligne SaintGermain-Poissy à travers la forêt, imposa leur remplacement, l'électrification par le fil aérien un moment envisagée fut repoussée par l'administration forestière (en raison des "dangers d'incendie dus aux étincelles de trolley !») et la Compagnie des chemins de fer de grande banlieue exploita la ligne avec trois automotrices Pieper à bogies. Ces voitures étaient en fait à transmission mixte. Le moteur de $66 \mathrm{~kW}$ commandait deux essieux par l'intermédiaire d'embrayages magnétiques. Une dynamo shunt calée sur l'arbre et reliée à une batterie d'accumulateurs pouvait être utilisée, soit comme génératrice, soit comme moteur, l'excédent d'énergie motrice du moteur thermique, emmagasiné sur parcours facile, étant restitué par les batteries pour aider au démarrage et sur profil difficile. Ces voitures pesant $22 \mathrm{t}$ et pouvant recevoir 60 voyageurs étaient capables de remorquer deux voitures de $10 \mathrm{t}$ sur rampe de $50 \mathrm{~mm}$. La vitesse moyenne haut-le-pied atteignait $25 \mathrm{~km} / \mathrm{h}$ 
pour une consommation de benzol (d'usine à gaz) guère meilleure que les Westinghouse, mais, à l'époque, moins coûteux que le pétrole importé.

Citons encore, côté des houillères du Nord, la curieuse automotrice Leroux construite par Fives-Lille et utilisée après 1911 sur Carvin-Libercourt: munie d'un moteur diesel avec démarrage à l'air comprimé, cette voiture de 29 t en charge, pouvant remorquer $33 \mathrm{t}$, fut détruite au cours de la guerre de 1914. Elle y avait d'ailleurs rejoint deux Westinghouse de 1911, à voie normale également, mais analogues à celle de Dinard-Saint-Briac.

La carrière de la plupart de ces précurseurs fut très courte. La guerre de 1914 raréfiant la main-d'œuvre et entravant l'entretien délicat de ces engins leur porta le coup de grâce, si bien qu'en 1920 il ne restait en service que l'automotrice Purrey du Loiret, deux automotrices Pieper sur Saint-Germain-Poissy, qui devaient finir peu glorieusement vingt années plus tard, débarrassées de leur équipement moteur compliqué, comme remorques incorporées aux trains à vapeur et, assez curieusement, l'automotrice Westinghouse du Dinard-Saint-Briac qui assura, en dépit de son robuste appétit, quelques services jusqu’à la fermeture de la ligne en 1930. Les trois voitures Westinghouse des Chemins de fer de grande banlieue qui avaient été envoyées à Lyon et munies de perches de prise de courant type tramway furent employées comme tracteurs à marchandises sur les voies de la Compagnie O.T.L., ce qui montre bien la souplesse d'adaptation des réseaux secondaires.

\section{L'« époque héroïque » 1922-1930 : vers l'autorail}

Après la guerre de 1914, la situation financière de la plupart des réseaux secondaires s'était largement détériorée. Ceux qui n’avaient pas été touchés par les destructions avaient, dans de nombreux cas, subi des réquisitions importantes. Le matériel envoyé en renfort, de provenance militaire, était usé et mal entretenu, et la concurrence routière débutante dépassait facilement les 15 à $20 \mathrm{~km} / \mathrm{h}$ des «tacots » campagnards. La seule parade qui parut possible fut de développer au maximum l'emploi d'automotrices, d'abord à essence, au moins pour assurer les trains les moins chargés, afin de récupérer le plus possible du trafic voyageurs qui échappait aux voies ferrées et de diminuer les frais d'exploitation, ainsi que l'expose une circulaire du 22 mai $1922 \mathrm{du}$ ministre des Travaux publics : «l'exploitation par train utilisant la traction à vapeur, si elle est avantageuse lorsque la charge des trains est suffisante, se trouve par contre presque toujours trop onéreuse aux heures de la journée où le trafic est faible. Cette constatation a conduit à l'étude de l'emploi de voitures automotrices à essence adaptées au rail, au besoin avec remorque. » 
Les premières réalisations originales furent l'œuvre de M. Tartary, administrateur de la Compagnie des tramways départementaux des Deux-Sèvres et de diverses autres compagnies. Dès 1921, des châssis de camions américains G.M.C. de 18 ch prélevés sur les stocks de guerre furent transformés dans les ateliers du réseau. Les modifications furent au demeurant assez peu importantes : raccourcissement des essieux, substitution de roues à boudins aux roues d'origine, l'empattement de 3,20 m ayant été jugé trop important pour les courbes de faible rayon ; le mécanisme de direction avait été conservé. Le volant devait permettre (du moins en théorie) au conducteur de faciliter l'inscription du véhicule dans les courbes! Un dispositif à ressort de rappel assurait la stabilité en ligne droite; des caisses de formes diverses achevaient de donner à "l'auto-rail », selon la dénomination du temps, un aspect assez extraordinaire. Ces engins ne comportant qu'un seul poste de conduite et ne pouvant être retournés au terminus sur les plaques tournantes normales, en raison de leur empattement, une solution ingénieuse avait été imaginée : une couronne de tôle munie d'un rebord était placée sur la voie en arrière de la voiture qui reculait et montait sur la couronne jusqu'à ce que l'essieu arrière occupe une position diamétrale, l'avant était soulevé et placé sur une roulette amovible. Le différentiel du châssis automobile ayant été conservé «l'auto-rail » était tourné en utilisant les roues arrières comme galets, la roulette se déplaçant sur un chemin constitué de plaques de tôle portatives posées sur le sol. Un homme seul pouvait, semble-t-il, effectuer la manœuvre en moins de dix minutes. Un tel « auto-rail » d'un poids de 2,2 t à vide seulement, mis en service sur la ligne de Château-Thierry à Verdelot (Chemin de fer du sud de l'Aisne) appartenant au groupe Tartary, atteignait une vitesse commerciale supérieure à $30 \mathrm{~km} / \mathrm{h}$ sur une ligne comportant des rampes de $3 \%$ en emmenant une vingtaine de voyageurs. La consommation d'essence ne dépassait pas 18 litres aux $100 \mathrm{~km}$. On pouvait alors espérer mieux encore que les autocars débutant sur les routes de l'époque.

Des véhicules de conception très semblable obtenus aussi à partir de G.M.C. furent construits par MM. Verney et mis en service à partir de 1922 sur divers réseaux (Finistère, Manche, Poitou, Seine-etMarne etc.). Dans beaucoup de cas, la capacité se révéla rapidement insuffisante et on attela des remorques très légères à un seul essieu pouvant recevoir les bagages ou même des voyageurs (avec un confort très aléatoire). 
Le système de retournement par plaque amovible, originalité du système, fut peu à peu remplacé dans les modèles plus récents par un dispositif de vérins permettant de soulever la voiture pour la faire pivoter autour d'un axe, reposant sur un socle amené au contact des rails, tout aussi ingénieux.

Vers la même époque, les Établissements Saurer construisirent quelques automotrices présentant aussi un aspect assez semblable à celui d'un autocar, mais obtenues à partir d'éléments neufs : elles furent mises en service sur divers réseaux (Dauphiné, Tramway du Pont-deBeauvoisin, Beaujolais, Ardèche, Indre-et-Loire, Corrèze...).

Mais ce furent surtout les Établissements De Dion Bouton qui, exploitant les brevets Tartary, réalisèrent à Puteaux les séries les plus importantes d'automotrices légères de conception « auto-rail » d'après 1920. Ces engins appartenaient à trois types principaux, tous différents et originaux :

les JA ou JM, pour voie étroite, étaient à quatre roues, l'essieu moteur arrière ne comportait pas de différentiel, mais on avait conservé le dispositif de fusées orientables pour faciliter l'inscription en courbe.

Les JL pour voie normale, également à deux essieux, dont un moteur, ne comportaient pas de dispositif de convergence.

Enfin, dans le type JB ou KG, on avait adopté un dispositif assez particulier: la caisse était portée à l'arrière par un essieu moteur et à l'avant par un avant-train articulé comportant quatre roues à axes indépendants disposées sur deux longerons mobiles articulés autour de l'essieu avant et pouvant, de plus, pivoter autour d'un axe vertical. Une biellette solidarisait ces deux longerons. De tels autorails circulèrent sur les réseaux des Côtes-du-Nord, de Châteaubriant à Erbray, de la Drôme, de l'Aude, etc. Les quelques cent engins JA, JM, JL, KG mis en service entre 1923 et 1933 eurent en général une carrière assez longue (certains assuraient encore quelques services jusqu'en 1956 sur les chemins de fer des Côtes-du-Nord). Un De Dion JM de ce dernier réseau a été conservé au musée de l'AMTUIR. Toutes ces automotrices étaient munies de moteurs à essence de 55 à 105 ch selon les types (soit 40 à $75 \mathrm{~kW}$ ) à l'arbre moteur.

Une esthétique plus « chemin de fer» mais de larges emprunts à la construction automobile caractérisaient les automotrices RenaultScemia, contemporaines des célèbres motrices $L$ des tramways parisiens, avec lesquelles elles avaient en commun la suspension à jumelles élastiques. Munies d'un moteur à essence de $45 \mathrm{ch}$ ou de 64 ch selon les 
types, et d'une boîte à quatre vitesses, elles assurèrent de bons services sur de nombreux réseaux (Tarn, Banlieue de Reims, Pas-de-Calais, embranchement de Marcoussis de l'Arpajonnais etc.). Elles roulaient encore après 1944 à Soissons et sur Corbeil-Milly-Étampes-Arpajon.

Certains réseaux désireux de posséder des autorails aux moindres frais et peu confiants dans le matériel automobile léger préférèrent transformer des voitures de leur parc par adjonction d'un moteur et d'une transmission mécanique : tels furent l'autorail Laborie du chemin de fer de Cormeilles à Glos-Montfort, qui présentait la particularité unique en 1924 d'être muni d'un gazogène à charbon de bois, et le prototype Berliet du réseau de Saône-et-Loire obtenu à partir d'une voiture à voyageurs à essieux convergents système de Rechter.

Nous citerons encore comme caractéristique de la construction type « chemin de fer » les automotrices Horme et Buire à deux essieux du Tarn-et-Garonne et du Lot-et-Garonne (moteur Ballot de 42 ou $54 \mathrm{ch}$ ) ou à bogies des Économiques des Charentes (moteur Latil de $64 \mathrm{ch})$.

La transmission électrique expérimentée avant 1914 n'était pas abandonnée et la firme Crochat s'en fit une spécialité. Dès 1923, une petite voiture était mise en service sur le réseau à voie de $0,60 \mathrm{~m}$ de Pithiviers à Thoury, où elle circula jusqu'en 1964 (voyageurs ou trains de service). Les caractéristiques en étaient les suivantes : longueur de la caisse $6,02 \mathrm{~m}$; poids $5,2 \mathrm{t}$; un moteur Aster à quatre cylindres de $19 \mathrm{ch}$, un moteur électrique de $10 \mathrm{~kW}$ actionnant un essieu. De nombreux autres modèles de conception analogue furent construits pour le Calvados, le Loir-et-Cher, le Vivarais, la Dordogne, la Drôme, etc. On peut constater l'extrême variété des modèles inventés et utilisés, toujours en avance sur les « grands réseaux ».

\section{L'autorail moderne : 1935-1960 environ}

L'évolution des automotrices à moteurs thermiques se poursuivait rapidement avec l'apparition de nouveaux moteurs et peu à peu les moteurs diesel, plus économiques, remplaçaient les moteurs à essence, et les caisses prirent un aspect plus moderne. Certains autorails continuaient à présenter un aspect fort semblable à celui d'un véhicule routier, au point que l'on jugea utile, aux Chemins de fer du Sud-Ouest, de placer à l'avant des autorails De Dion un panneau lumineux figurant ... une locomotive, indiquant qu'il s'agissait bien d'un engin sur rail et non d'un autocar, ceci pour éviter toute confusion de la part des automobilistes voisinant avec le chemin de fer lorsqu'il était tracé en accotement de la route. 
Une mention particulière doit être faite pour cette période des très nombreux autorails Billard à bogies, mis en service à partir de 1935, et dont la silhouette si particulière est devenue en peu d'années un « élément du décor» de la plupart des réseaux secondaires. Caractérisés par un bogie moteur portant le moteur lui-même, un centre de gravité très bas et une suspension particulièrement bien adaptée aux voies médiocres, ils ont permis à un grand nombre de réseaux de survivre jusqu'à nos jours. Ces engins d'une capacité suffisante (32 places assises, 15 debout) et légers (poids à vide $7 \mathrm{t}$ pour les plus petits modèles), sont susceptibles d'atteindre des vitesses de pointe de $80 \mathrm{~km} / \mathrm{h}$ et de tirer le cas échéant une remorque de même conception. Ils ont été munis de moteurs très divers (Unic, Berliet, C.L.M., Man, Willème) d'une puissance variant de 80 à $210 \mathrm{ch}(50$ à $150 \mathrm{~kW})$. De nombreux exemplaires ont circulé sur la plupart des réseaux secondaires (réseau breton, Corrèze, Corse, Vivarais, Lozère).

Contemporaines des autorails Billard, et mises en service de 1934 à 1938 sur divers réseaux (Anjou, Vallée de Celles, Économiques des Charentes, Morbihan, Provence), les automotrices diesel-électriques Brissonneau et Lotz étaient caractérisées par une conduite aisée et souple due à leur transmission. La régulation de la puissance était obtenue par la variation de vitesse du moteur diesel et le réglage automatique de la génératrice par contacteurs vibrants. L'entretien délicat de la partie électrique pendant la guerre a abrégé dans beaucoup de cas leur carrière, en dépit de performances très honorables. Les autorails doubles du réseau du Var, des Chemins de fer de Provence, munis de deux moteurs Berliet de $150 \mathrm{ch}$ (total $220 \mathrm{~kW}$ ) pouvaient atteindre $90 \mathrm{~km} / \mathrm{h}$ (poids en charge $37 \mathrm{t}, 60$ places assises). Ils ont repris du service depuis la fermeture du réseau du littoral de Provence sur les lignes espagnoles de Santander à Bilbao, tout aussi littorales mais heureusement conservées.

Cependant, les grands réseaux et les constructeurs, reprenant avec conviction, à leur tour, le développement des autorails après 1935, avec des séries plus importantes, les autorails les plus récemment construits pour les réseaux secondaires tendent de plus en plus à ressembler au matériel de grand réseau : caisses à grande capacité montées sur bogies, moteurs diesel, transmissions mécaniques, et cela jusqu'après la guerre 1939-1944 et encore plus de nos jours.

Parmi les matériels plus modernes, nous pouvons citer les autorails Renault A B H mis en service de 1935 à 1948 sur divers réseaux à voie métrique (Corse, Provence, Côtes-du-Nord). Ces engins sont munis de moteurs de 265 à $300 \mathrm{ch}$ (200 à $220 \mathrm{~kW}$ ) et dérivent directement des 
autorails Renault A B J, alors très répandus sur la $\mathrm{SNCF}$, avec les mêmes moteurs. Après plus de 65 ans de service, Nice-Digne peut être fière de pouvoir en faire encore circuler.

En 1946-1948 le réseau breton a reçu six autorails De Dion de $180 \mathrm{ch}$ (140 kW, à bogies), d'une capacité de 51 places assises, susceptibles d'atteindre $70 \mathrm{~km} / \mathrm{h}$ (type OC) qui vont faire souche. Mutés sur le Blanc-Argent en 1967, nous avons pu en voir un exemplaire fort bien restauré au dépôt de Romorantin.

Citons encore les autorails S.C.F. (Verney) de 180 et 150 ch des Chemins de fer de la Corrèze et du Blanc à Argent (fig. 4) mis en service en 1950-1951. D’un poids de 24,4 t en charge et d'une capacité de 73 voyageurs, ces voitures ont atteint $96 \mathrm{~km} / \mathrm{h}$ en palier. Leur service a été assez fructueux puisqu'ils ont pu ensuite bénéficier d'une modernisation à «mi-vie », en 1983-1984. Modernisés aussi côté moteurs, ils passent à 113 kW (Poyaud) et 173 kW (MAN) en 1994.

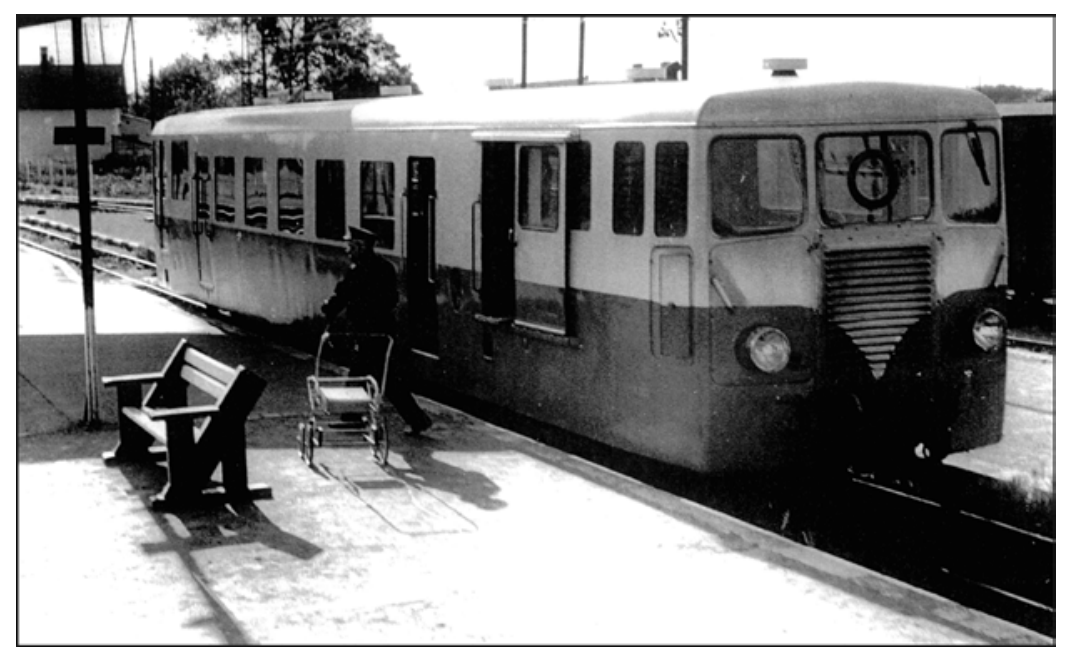

Figure 4 : Un des autorails du Blanc-Argent type Verney de 1948 à Gièvres SNCF.

Coll. Y. Machefert-Tassin, cl. d.r.

Enfin, parmi les autres réalisations de cette période, nous pourrons encore mentionner les autorails légers à voie normale étudiés par la Compagnie de chemins de fer départementaux. A deux essieux, d'une capacité de 60 places environ, pesant 8 t et munis d'un moteur Panhard de $80 \mathrm{ch}$, ces engins peuvent être considérés comme de véritables cars sur rail, mais non comme des innovations. 


\section{L'éclipse puis la renaissance d'après 1990}

Dès la fin des années 1950, malgré les progrès techniques alors disponibles et réalisés pour certains matériels roulants, les réseaux secondaires français s'amenuisent ou disparaissent définitivement sans aucun scrupule prospectif régional ou local des pouvoirs publics exclusivement dirigés vers la route. Il n'est donc pas étonnant que, pendant près de 40 ans, aucun progrès, apparaissant du côté des grands réseaux, n’ait de répercussion en chemins de fer « secondaires » ou « régionaux ». Ce qui ne veut pas dire que des progrès ne soient pas accomplis sur voie normale ou étroite, en automotrices ou autorails d'autres contrées, où les constructeurs français exportent largement des modèles adaptés aux besoins locaux - l'Oferom, pour les chemins de fer francophones d'outremer participe activement à l'élaboration des nouvelles locomotives ou autorails de puissance de plus en plus élevée pour ces réseaux à voie métrique le plus généralement et qui sont souvent essayés préalablement en France sur les lignes restantes les mieux armées (La Mure par exemple).

Par ailleurs, la concentration des moyens industriels de production de matériel roulant ferroviaire en quelques compagnies européennes ou multinationales permet aussi d'heureuses interférences de techniques nouvelles autrefois indépendantes, séparées, ou concurrentes. Les besoins nouveaux, tant pour des réseaux périurbains que régionaux font alors apparaitre la nécessité, pour chaque groupement de constructeurs, de présenter à des clients devenus multiples et plus ouverts à la concurrence internationale des solutions spécifiques adaptées à partir d'une gamme de produits existants ou à développer. C'est ainsi que naissent, en automotrices et autorails, notamment, les rames dites modulaires, combinant, à l'exemple des nouveaux tramways de cette décennie, des éléments moteurs quelle que soit la source d'énergie (électrique ou dieselélectrique) et des éléments allégés poussés ou remorqués, articulés ou non, autour du centre moteur. C'est ainsi qu'apparaissent, à partir de la Suisse, les GTW (Gelenktriebwagen ou rames articulées à motrice centrale) de Stadler-AD Tranz (Bombardier) réalisables en une dizaine de combinaisons, pour n'importe quel écartement de voie ou gabarit disponible et pour n'importe quel pays d'Europe (en dernier lieu Slovaquie et Grèce).

Par ailleurs, et un peu plus classiquement, à partir du savoir-faire des établissements industriels de Soulé à Bagnères de Bigorre qui avaient produit les A2E - CFTA Bretagne et X97050 Corse de 1987 à 1992, les C.F.D. produisent de nouveaux autorails, aux normes proches de celles de la SNCF, adaptés à la voie métrique, tant attendus à Romorantin 
pour la ligne B.A., même réduite à Valençay-Salbris. On peut donc avoir encore bon espoir pour l'ouverture, ou la réouverture de lignes dites secondaires : le matériel roulant le meilleur y sera disponible.

\section{Conclusion}

Après avoir rapidement passé en revue l'évolution technique prémonitoire, puis l'action de précurseur et les apports en tous domaines des automotrices des chemins de fer secondaires français, il nous reste à constater les résultats.

L'utilisation intensive des autorails a permis, dans la plupart des cas, d'aboutir à une séparation totale des services voyageurs et marchandises et de supprimer les rebutants « trains mixtes » des lignes à faible trafic. De plus, outre les gains de temps et de vitesse entre vapeur et tractions nouvelles, en rapport de deux à un, surtout sur les lignes les plus difficiles, l'économie était énorme et certains la pensaient salvatrice.

Par exemple, le prix de revient du km-autorail était évalué en 1939 à environ 2,50 F pour un autorail métrique d'époque, équipé d'un moteur diesel de $147 \mathrm{~kW}$. L'économie était déjà de 50 à $60 \%$ par rapport à la traction vapeur, malgré le prix alors élevé des produits pétroliers.

La mise en service des autorails permit quelquefois des augmentations spectaculaires du trafic : $314 \%$ pour la ligne du littoral (Toulon à Saint-Raphaël) des Chemins de fer de Provence, après substitution des rames diesel-électriques Brissonneau et Lotz aux trains à vapeur. Malheureusement, comme en ce cas particulier, on n'a pas compris l'intérêt de cette évolution pour améliorer aussi l'implantation des lignes et les rendre indépendantes des routes. Il faut bien reconnaitre cependant que, dans certains cas, l'autorail, surtout à ses débuts, apparaissait sous un jour peu séduisant : caisse exiguë incapable d'absorber les surcharges, moteurs malodorants et bruyants... Nous nous souvenons, avec d'autres, d'un interminable voyage de près de deux heures à travers la forêt de la Coubre, près de Royan, où l'odeur d'essence mal raffinée de la petite automotrice cahotante (Campagne, de 1924), triomphait facilement de la senteur des pins.

Compte tenu des progrès effectués qui ont abouti de nos jours à la réalisation d'engins confortables et rapides, le bilan de l'emploi des automotrices électriques et des autorails reste très positif et il faut reconnaitre que les chemins de fer secondaires peuvent, dans le domaine de la conception et de l'utilisation des automotrices, être considérés comme des précurseurs. Ayant su, en métropole, éviter un certain nombre d'expériences coûteuses, ils ont mis en service, dix à vingt ans avant 
les grands réseaux, des engins dans l'ensemble bien étudiés et bien adaptés au service demandé, dont la longévité fut pour beaucoup une preuve de leur réussite, ce qui favorise aujourd'hui les lignes touristiques.

Au détriment, certes, d'un certain pittoresque, la substitution d'automotrices modernes à la plupart des trains a permis à beaucoup de réseaux des survies de plus de vingt ans et, pour certains d'entre eux, de durer, en luttant avec succès contre la concurrence routière. Cela reste vrai même en France de nos jours, en des cas spécifiques, et quel que soit le type de traction utilisé.

\section{Note}

1- Voir :

- L'Histoire des chemins de fer en France, Paris, Les Presses Modernes, 1963 ;

- Autour des chemins de fer français, Paris, Les Presses Modernes, 1966 ;

- Histoire de la traction électrique, Paris, La Vie du rail, t. 1, 1980 ; t. 2, 1986 ;

- Histoire des autorails en France, Paris, La Vie du rail, t. 1, 1992 ; t. 2, 1994 ; t. 3, 1997;

- Le Patrimoine de la SNCF et des chemins de fer français, tomes 1 et 2, Charenton, Flohic, 1999-2000 ;

- «L'Électrification des chemins de fer secondaires en France : une occasion manquée », in «Électricité et chemins de fer : cent ans de progrès ferroviaire par l'électricité », Xं coll. de l'AHEF / 5 coll. de l'AHICF, Paris, 1995, Revue d'bistoire des chemins de fer hors série, 5, 1996 ;

- Les Petits Trains de jadis, Éditions du Cabri, tomes 1 à 9. 